Pacific

Journal of

Mathematics

\title{
DEMYSTIFYING A DIVISIBILITY PROPERTY OF THE KOSTANT PARTITION FUNCTION
}

\author{
KAROLA MÉSZÁROS
}




\title{
DEMYSTIFYING A DIVISIBILITY PROPERTY OF THE KOSTANT PARTITION FUNCTION
}

\author{
KAROLA MÉSZÁROS
}

\begin{abstract}
We prove a family of identities connected to a divisibility property of the Kostant partition function. A special case of these identities first appeared in a paper of Baldoni and Vergne. To prove their identities, Baldoni and Vergne used residue techniques, and called the resulting divisibility property "mysterious." Our proofs are entirely combinatorial and provide a natural explanation for why divisibility occurs, both in the Baldoni and Vergne identities and in their generalizations.
\end{abstract}

\section{Introduction}

The objective of this paper is to provide a natural combinatorial explanation of a divisibility property of the Kostant partition function. The question of evaluating Kostant partition functions has been the subject of much interest, without a satisfactory combinatorial answer. To mention perhaps the most famous such case: it is known that

$$
K_{A_{n}^{+}}\left(1,2, \ldots, n,-\left(\begin{array}{c}
n+1 \\
2
\end{array}\right)\right)=\prod_{k=1}^{n} C_{k}, \quad \text { where } C_{k}=\frac{1}{k+1}\left(\begin{array}{c}
2 k \\
k
\end{array}\right)
$$

denotes the Catalan numbers, yet there is no combinatorial proof of this identity! While endowed with combinatorial meaning, Kostant partition functions were introduced in and are a vital part of representation theory: weight multiplicities and tensor product multiplicities can be expressed in terms of the Kostant partition function. Kostant partition functions also come up in toric geometry and analytic residue theory.

Given the lack of understanding of the evaluation of the Kostant partition function, it seems a worthy proposition to provide a simple explanation for certain of its divisibility properties. We explore divisibility properties of Kostant partition functions of types $A_{n}$ and $C_{n+1}$, noting that such properties in types $B_{n+1}$ and $D_{n+1}$ are easy consequences of the type $C_{n+1}$ case. A significant part of the type $A_{n}$

The author is supported by a National Science Foundation Postdoctoral Research Fellowship. MSC2010: 05E10.

Keywords: Kostant partition function, divisibility, flow. 
family of identities we study first appeared in [Baldoni and Vergne 2008], where the authors prove the identities using residues, and where they call the divisibility property "mysterious." It is our hope that the combinatorial argument we provide successfully demystifies the divisibility property of the Kostant partition function and provides a natural explanation for why things happen the way they do.

The outline of the paper is as follows. In Section 2 we define Kostant partition functions of type $A_{n}$ and prove a family of identities, including the Baldoni-Vergne identities, combinatorially. Our proof is bijective, and as such it also yields an affirmative answer to a question of Stanley [2000] regarding a possible bijective proof of a special case of the Baldoni-Vergne identities. In Section 3 we define Kostant partition functions of type $C_{n+1}$, relate them to flows, and show how to modify our proof of the identities from Section 2 to obtain their analogues for type $C_{n+1}$.

\section{A family of Kostant partition function identities}

In this section we prove a family of Kostant partition function identities exhibiting divisibility properties. We start by proving Baldoni-Vergne identities, our proof of which clearly points to several generalizations of these identities. We provide some of these generalizations in this section, and some in the next.

The Baldoni-Vergne identities. Before stating the Baldoni-Vergne identities, we need a few definitions. Throughout this section the graphs $G$ we consider are on the vertex set $[n+1]$, possibly with multiple edges, but no loops. Denote by $m_{i j}$ the multiplicity of edge $(i, j), i<j$, in $G$. To each edge $(i, j), i<j$, of $G$, associate the positive type $A_{n}$ root $e_{i}-e_{j}$, where $e_{i}$ is the $i$-th standard basis vector. Let $\left\{\left\{\alpha_{1}, \ldots, \alpha_{N}\right\}\right\}$ be the multiset of vectors corresponding to the multiset of edges of $G$ as described above. Note that $N=\sum_{1 \leq i<j \leq n+1} m_{i j}$.

The Kostant partition function $K_{G}$ evaluated at the vector $\boldsymbol{a} \in \mathbb{Z}^{n+1}$ is defined as

$$
K_{G}(\boldsymbol{a})=\#\left\{\left(b_{i}\right)_{i \in[N]} \mid \sum_{i \in[N]} b_{i} \alpha_{i}=\boldsymbol{a} \text { and } b_{i} \in \mathbb{Z}_{\geq 0}\right\} .
$$

That is, $K_{G}(\boldsymbol{a})$ is the number of ways to write the vector $\boldsymbol{a}$ as a nonnegative linear combination of the positive type $A_{n}$ roots corresponding to the edges of $G$, without regard to order. Note that in order for $K_{G}(\boldsymbol{a})$ to be nonzero, the partial sums of the coordinates of $\boldsymbol{a}$ have to satisfy $a_{1}+\cdots+a_{i} \geq 0, i \in[n]$, and $a_{1}+\cdots+a_{n+1}=0$.

We now proceed to state and prove Theorem 1, which first appeared in [Baldoni and Vergne 2008]. Baldoni and Vergne gave a proof of it using residues, and called the result "mysterious". We provide a natural combinatorial explanation of the result. Our explanation also answers a question of Stanley [2000] in the 
affirmative, regarding a possible bijective proof of a special case of the BaldoniVergne identities.

For brevity, we write $G-e$, or $G-\left\{e_{1}, \ldots, e_{k}\right\}$, to mean a graph obtained from $G$ with the edge $e$, or the edges $e_{1}, \ldots, e_{k}$, deleted.

Theorem 1 [Baldoni and Vergne 2008]. Given a connected graph $G$ on the vertex set $[n+1]$ with $m_{n-1, n}=m_{n-1, n+1}=m_{n, n+1}=1$, and such that

$$
\frac{m_{j, n-1}+m_{j, n}+m_{j, n+1}}{m_{j, n-1}}=c \quad \text { for all } j \in[n-2],
$$

for some constant $c$ independent of $j$, we have

$$
K_{G}(\boldsymbol{a})=\left(\frac{a_{1}+\cdots+a_{n-2}}{c}+a_{n-1}+1\right) K_{G-(n-1, n)}(\boldsymbol{a}),
$$

for any $\boldsymbol{a}=\left(a_{1}, \ldots, a_{n},-\sum_{i=1}^{n} a_{i}\right) \in \mathbb{Z}^{n+1}$.

Before proceeding to the formal proof of Theorem 1 we outline it, to fully expose the underlying combinatorics. We introduce the notation

$$
Q(\boldsymbol{a}):=\frac{a_{1}+\cdots+a_{n-2}}{c}+a_{n-1}+1
$$

for the factor in (2). Rephrasing Equation (1), $K_{G}(\boldsymbol{a})$ counts the number of flows $\boldsymbol{f}_{G}=\left(b_{i}\right)_{i \in N}$ on $G$ satisfying

$$
\sum_{i \in[N]} b_{i} \alpha_{i}=\boldsymbol{a} \quad \text { and } \quad b_{i} \in \mathbb{Z}_{\geq 0} .
$$

In the proof of Theorem 1, we introduce the concept of partial flows $f_{H}$, about which we prove two key statements:

- The elements of the set of partial flows are in bijection with the flows on $G-(n-1, n)$ that the Kostant partition function $K_{G-(n-1, n)}(\boldsymbol{a})$ counts. That is,

$$
\text { \# partial flows }=K_{G-(n-1, n)}(\boldsymbol{a}) \text {. }
$$

- The elements of the multiset of partial flows $\boldsymbol{f}_{H}$ - whose cardinality is $Q(\boldsymbol{a})$ times the cardinality of the set of partial flows - are in bijection with the flows on $G$ that the Kostant partition function $K_{G}(\boldsymbol{a})$ counts. That is,

$$
Q(\boldsymbol{a}) \text { (\# partial flows) }=K_{G}(\boldsymbol{a}) .
$$

From these two statements we see that the two Kostant partition functions $K_{G}(\boldsymbol{a})$ and $K_{G-(n-1, n)}(\boldsymbol{a})$ are connected by

$$
K_{G}(\boldsymbol{a})=Q(\boldsymbol{a}) K_{G-(n-1, n)}(\boldsymbol{a}),
$$

which is Equation (2). 
Proof of Theorem 1. Let $\left\{\left\{\alpha_{1}, \ldots, \alpha_{N}\right\}\right\}$ be the multiset of vectors corresponding to the edges of $G$. Let $\alpha_{N}=e_{n-1}-e_{n}, \alpha_{N-1}=e_{n-1}-e_{n+1}$, and $\alpha_{N-2}=e_{n}-e_{n+1}$. Then (2) can be rewritten as

$$
\#\left\{\left(b_{i}\right)_{i \in[N]} \mid \sum_{i=1}^{N} b_{i} \alpha_{i}=\boldsymbol{a}\right\}=Q(\boldsymbol{a}) \#\left\{\left(b_{i}\right)_{i \in[N-1]} \mid \sum_{i=1}^{N-1} b_{i} \alpha_{i}=\boldsymbol{a}\right\},
$$

where $Q(\boldsymbol{a})$ is defined in (3).

We proceed to prove the equality (4) bijectively. The key concept we use is that of a partial flow, which we now define.

Consider a flow $\boldsymbol{f}_{H}=\left(b_{i}\right)_{i \in[N-3]}\left(b_{i} \in \mathbb{Z}_{\geq 0}\right)$ on the edges of the graph

$$
H:=G-\{(n-1, n),(n-1, n+1),(n, n+1)\} .
$$

We call $\boldsymbol{f}_{H}$ partial if

$$
\sum_{i=1}^{N-3} b_{i} \alpha_{i}=\left(a_{1}, \ldots, a_{n-2}, x_{n-1}, x_{n}, x_{n+1}\right),
$$

for some $x_{n-1}, x_{n}, x_{n+1} \in \mathbb{Z}$ satisfying $x_{n-1} \leq a_{n-1}$ and $x_{n} \leq a_{n}$.

Note that a given partial flow $\boldsymbol{f}_{H}=\left(b_{i}\right)_{i \in[N-3]}$ can be extended uniquely to a flow $\boldsymbol{f}_{G-\{(n-1, n)\}}=\left(b_{i}\right)_{i \in[N-1]}\left(b_{i} \in \mathbb{Z}_{\geq 0}\right)$ on $G-\{(n-1, n)\}$ such that $\sum_{i=1}^{N-1} b_{i} \alpha_{i}=\boldsymbol{a}$. Furthermore, each such flow arises from a uniquely determined partial flow. Denote by $f$ the map that takes a partial flow $\boldsymbol{f}_{H}$ into a flow $\boldsymbol{f}_{G-\{(n-1, n)\}}$ as just described. See Figure 1 for an example. The observations above imply:

Claim 1. The map $f$ establishes a bijection between the set of partial flows $\boldsymbol{f}_{H}$ on $H$ and the set of flows on $G-\{(n-1, n)\}$ such that $\sum_{i=1}^{N-1} b_{i} \alpha_{i}=\boldsymbol{a}$. In symbols,

$$
\#\left\{\left(b_{i}\right)_{i \in[N-1]} \mid \sum_{i=1}^{N-1} b_{i} \alpha_{i}=\boldsymbol{a}\right\}=\sum_{f_{H}} 1,
$$

where the summation runs over all partial flows $\boldsymbol{f}_{H}$.
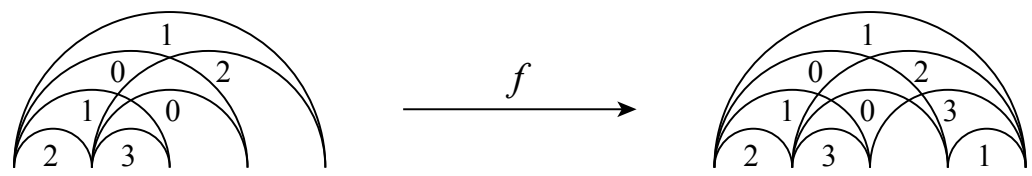

Figure 1. Here $G$ is the complete graph on 5 vertices and $\boldsymbol{a}=$ $(4,3,-1,1,-7)$. The flows are written immediately below the corresponding edges. On the left is a partial flow $f_{H}$ and on the right is its image under $f$. 
Note that the left-hand side of (6) is the same as the cardinality on the right-hand side of (4).

Given a partial flow $f_{H}$, denote by $Y_{i}\left(f_{H}\right)$, for $i \in\{n-1, n, n+1\}$, the total inflow into vertex $i \in\{n-1, n, n+1\}$ in $H$, that is, the sum of all the flows $b_{i}$ on edges of $H$ incident to $i \in\{n-1, n, n+1\}$. Note that a partial flow $f_{H}$ can be extended in $Y_{n-1}\left(\boldsymbol{f}_{H}\right)+a_{n-1}+1$ ways to a flow $\boldsymbol{f}_{G}=\left(b_{i}\right)_{i \in[N]}\left(b_{i} \in \mathbb{Z}_{\geq 0}\right)$ of $G$ such that $\sum_{i=1}^{N} b_{i} \alpha_{i}=\boldsymbol{a}$. Furthermore, given a flow $\boldsymbol{f}_{G}=\left(b_{i}\right)_{i \in[N]}\left(b_{i} \in \mathbb{Z}_{\geq 0}\right)$ such that $\sum_{i=1}^{N} b_{i} \alpha_{i}=\boldsymbol{a}$, there is a unique partial flow $\boldsymbol{f}_{H}=\left(b_{i}\right)_{i \in[N-3]}$ from which it can be obtained. Therefore, the above establishes a map $g$ which is a bijection between the multiset of partial flows $\mathcal{M}$ such that each partial flow $f_{H}$ appears exactly $Y_{n-1}\left(\boldsymbol{f}_{H}\right)+a_{n-1}+1$ times in $\mathcal{M}$, and the (multi)set of flows $\boldsymbol{f}_{G}=\left(b_{i}\right)_{i \in[N]}$ $\left(b_{i} \in \mathbb{Z}_{\geq 0}\right)$ on $G$ such that $\sum_{i=1}^{N} b_{i} \alpha_{i}=\boldsymbol{a}$. See Figure 2 for an example. We thus obtain

$$
\#\left\{\left(b_{i}\right)_{i \in[N]} \mid \sum_{i=1}^{N} b_{i} \alpha_{i}=\boldsymbol{a}\right\}=\sum_{f_{H}}\left(Y_{n-1}\left(f_{H}\right)+a_{n-1}+1\right)=\# \mathcal{M},
$$

where the second summation runs over the set of partial flows $f_{H}$.
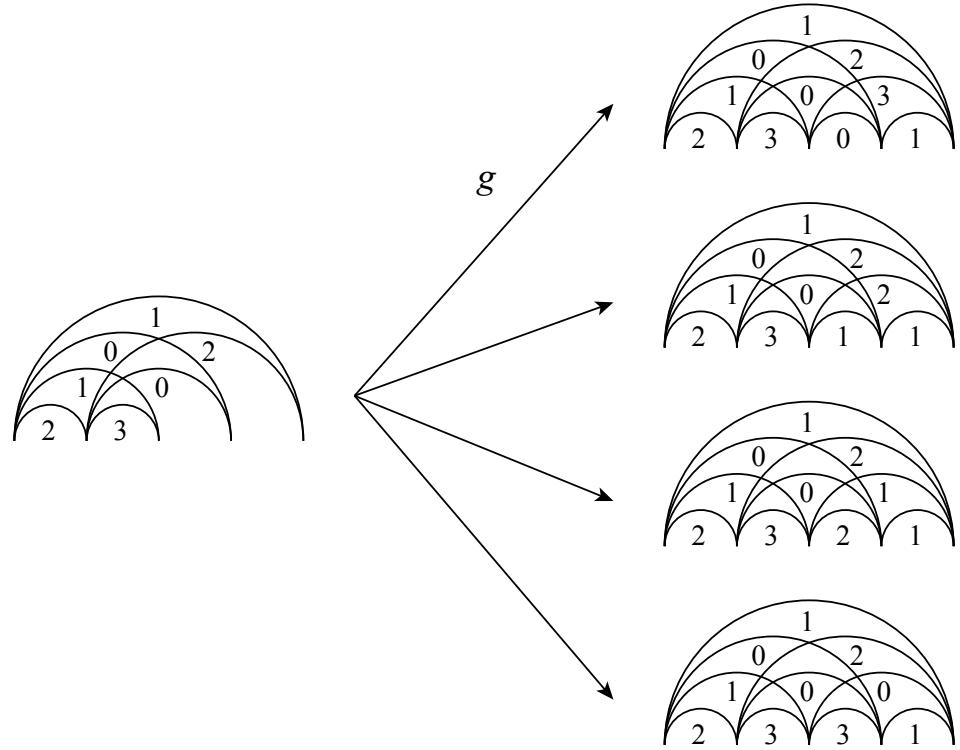

Figure 2. Again $G$ is the complete graph on 5 vertices and $\boldsymbol{a}=$ $(4,3,-1,1,-7)$. The flows are written immediately below the corresponding edges. On the left is a partial flow $f_{H}$ and on the right are the $Y_{n-1}\left(f_{H}\right)+a_{n-1}+1=4$ images under $g$ of the 4 copies of $f_{H}$ appearing in $M$. 
Claim 2. The map g just described establishes a bijection between the multiset $M$ of partial flows $\boldsymbol{f}_{H}=\left(b_{i}\right)_{i \in[N-3]}$ and the (multi)set of flows $\boldsymbol{f}_{G}=\left(b_{i}\right)_{i \in[N]}\left(b_{i} \in \mathbb{Z}_{\geq 0}\right)$ on $G$ such that $\sum_{i=1}^{N} b_{i} \alpha_{i}=\boldsymbol{a}$. Moreover,

$$
\# M=Q(\boldsymbol{a}) \sum_{f_{H}} 1,
$$

where the summation runs over all partial flows $\boldsymbol{f}_{H}$.

We already showed that $g$ is a bijection; there remains to show (8). Now, by assumption we have

$$
\frac{m_{j, n-1}+m_{j, r}+m_{j, n+1}}{m_{j, n-1}}=c,
$$

where $c$ is independent of $j \in[n-2]$; hence

$$
\begin{aligned}
c \sum_{f_{H}} Y_{n-1}\left(f_{H}\right) & =\sum_{f_{H}}\left(Y_{n-1}\left(f_{H}\right)+Y_{n}\left(f_{H}\right)+Y_{n+1}\left(f_{H}\right)\right) \\
& =\sum_{f_{H}}\left(a_{1}+\cdots+a_{n-2}\right),
\end{aligned}
$$

that is,

$$
\sum_{f_{H}} Y_{n-1}\left(f_{H}\right)=\sum_{f_{H}} \frac{a_{1}+\cdots+a_{n-2}}{c} .
$$

Adding $a_{n-1}+1$ to both summands and recalling (3) and the second equality in (7) yields (8), completing the proof of Claim 2.

In light of (4) it is clear that Claims 1 and 2 together provide a bijective proof of Theorem 1. In terms of equations this can also be seen as follows. Rewrite (7) as

$$
\begin{aligned}
\#\left\{\left(b_{i}\right)_{i \in[N]} \mid \sum_{i=1}^{N} b_{i} \alpha_{i}=\boldsymbol{a}\right\} & =Q(\boldsymbol{a}) \sum_{\boldsymbol{f}_{H}} 1 \\
& =Q(\boldsymbol{a}) \#\left\{\left(b_{i}\right)_{i \in[N-1]} \mid \sum_{i=1}^{N-1} b_{i} \alpha_{i}=\boldsymbol{a}\right\},
\end{aligned}
$$

where the first equality uses (7) and (8), and the second equality uses (6).

Further Kostant partition function identities. Several generalizations are suggested by our proof of the Baldoni-Vergne identities. Here we present an immediate one, Theorem 2; to ensure clarity we include its proof, which follows that of Theorem 1. For brevity, we do not explicitly state the analogues of Claims 1 and 2 from the proof of Theorem 1, though it is easy to obtain them from our proof. In the next section we present further generalizations of other types, but we omit the proofs. 
Theorem 2. Let $G$ be a connected graph on the vertex set $[n+1]$. Given $k \leq n$, suppose the graph $S=(V(S), E(S))$ defined by

$$
V(S)=\{k, k+1, \ldots, n, n+1\} \subset[n+1]
$$

and

$$
E(S)=\{(i, j) \in E(G) \mid i<j, i \in V(S)\}
$$

satisfies these conditions: that

- $\operatorname{outdeg}_{S}(j)=2$ and $\operatorname{indeg}_{S}(j)=0$, for some $k \leq j \leq n$, and $\operatorname{outdeg}_{S}(i)=1$ for $k \leq i \leq n, i \neq j$.

- For some constant c independent of $l$,

$$
\frac{\sum_{i=k}^{n+1} m_{l, i}}{m_{l, j}}=c \quad \text { for all } l \in[k-1] .
$$

Let $(j, z)$ be one of the outgoing edges from $j$ in $S$. Then

$$
K_{G}(\boldsymbol{a})=Q^{\prime}(\boldsymbol{a}) K_{G-(j, z)}(\boldsymbol{a}),
$$

for any $\boldsymbol{a}=\left(a_{1}, \ldots, a_{n},-\sum_{i=1}^{n} a_{i}\right) \in \mathbb{Z}^{n+1}$, where $Q^{\prime}(\boldsymbol{a})=\frac{a_{1}+\cdots+a_{k-1}}{c}+a_{j}+1$.

The most important cases of Theorem 2 are for $k \geq n-1$, since for $k<n-1$ several of the edges of $G$ can be "contracted" and reduced to the case $k \geq n-1$. For $k \geq n-1$ we obtain four interesting cases from Theorem 2 depending on the form of the graph $S$ and the edge $(j, z)$. If we take

$$
V(S)=\{n-1, n, n+1\} \quad \text { and } \quad E(S)=\{(n-1, n),(n-1, n+1),(n, n+1)\}
$$

and $(j, z)=(n-1, n)$, then Theorem 2 specializes to the original Baldoni-Vergne identities, while other choices of $S$ and $(j, z)$ lead to new identities.

Proof of Theorem 2. Let $\left\{\left\{\alpha_{1}, \ldots, \alpha_{N}\right\}\right\}$ be the multiset of vectors corresponding to the edges of $G$ such that the multiset of vectors corresponding to the edges of $S$ are $\left\{\left\{\alpha_{N}-(n+1-k), \ldots, \alpha_{N}\right\}\right\}$. Also, let $\alpha_{N}$ correspond to the edge $(j, z)$.

Consider a flow $\boldsymbol{f}_{H}=\left(b_{i}\right)_{i \in[N-(n+2-k)]}\left(b_{i} \in \mathbb{Z}_{\geq 0}\right)$ of the edges of the graph $H:=([n+1], E(G) \backslash E(S))$. We call $f_{H}$ partial if

$$
\sum_{i=1}^{N-(n+2-k)} b_{i} \alpha_{i}=\left(a_{1}, \ldots, a_{k-1}, x_{k}, x_{k+1}, \ldots, x_{n+1}\right),
$$

for some $x_{i} \in \mathbb{Z}, k \leq i \leq n+1$ satisfying $x_{i} \leq a_{i}$, for $k \leq i \leq n$. 
A given partial flow $\boldsymbol{f}_{H}=\left(b_{i}\right)_{i \in[N-(n+2-k)]}$, it can be extended uniquely to a flow $\boldsymbol{f}_{G-\{(j, z)\}}=\left(b_{i}\right)_{i \in[N-1]}\left(b_{i} \in \mathbb{Z}_{\geq 0}\right)$ on $G-\{(j, z)\}$ such that $\sum_{i=1}^{N-1} b_{i} \alpha_{i}=\boldsymbol{a}$. Furthermore, this correspondence is a bijection. Therefore,

$$
\#\left\{\left(b_{i}\right)_{i \in[N-1]} \mid \sum_{i=1}^{N-1} b_{i} \alpha_{i}=\boldsymbol{a}\right\}=\sum_{f_{H}} 1,
$$

where the summation runs over all partial flows $\boldsymbol{f}_{H}$.

Given a partial flow $\boldsymbol{f}_{H}$ in $H$, we denote by $Y_{i}\left(\boldsymbol{f}_{H}\right)$, where $i \in\{k, k+1, \ldots, n+1\}$, the total inflow into vertex $i$, that is, the sum of all the flows $b_{i}$ on edges of $H$ incident to $i$. The partial flow $\boldsymbol{f}_{H}$ can be extended in $Y_{j}\left(f_{H}\right)+a_{j}+1$ ways to a flow $\boldsymbol{f}_{G}=\left(b_{i}\right)_{i \in[N]}\left(b_{i} \in \mathbb{Z}_{\geq 0}\right)$ of $G$ such that $\sum_{i=1}^{N} b_{i} \alpha_{i}=\boldsymbol{a}$. Furthermore, given a flow $\boldsymbol{f}_{G}=\left(b_{i}\right)_{i \in[N]}\left(b_{i} \in \mathbb{Z}_{\geq 0}\right)$ such that $\sum_{i=1}^{N} b_{i} \alpha_{i}=\boldsymbol{a}$, there is a unique partial flow $\boldsymbol{f}_{H}=\left(b_{i}\right)_{i \in[N-(n+2-k)]}$ from which it can be obtained. Therefore,

$$
\#\left\{\left(b_{i}\right)_{i \in[N]} \mid \sum_{i=1}^{N} b_{i} \alpha_{i}=\boldsymbol{a}\right\}=\sum_{\boldsymbol{f}_{H}}\left(Y_{j}\left(\boldsymbol{f}_{H}\right)+a_{j}+1\right),
$$

where the summation runs over all partial flows $\boldsymbol{f}_{H}$.

By assumption, we have

$$
\frac{\sum_{i=k}^{n+1} m_{l, i}}{m_{l, j}}=c,
$$

where $c$ is independent of $l \in[k-1]$; hence

$$
c \sum_{f_{H}} Y_{j}\left(f_{H}\right)=\sum_{f_{H}} \sum_{i=k}^{n+1} Y_{i}\left(f_{H}\right)=\sum_{f_{H}}\left(a_{1}+\cdots+a_{k-1}\right),
$$

that is,

$$
\sum_{f_{H}} Y_{j}\left(f_{H}\right)=\sum_{f_{H}} \frac{a_{1}+\cdots+a_{k-1}}{c} .
$$

Thus, (15) can be rewritten as

$$
\begin{aligned}
\#\left\{\left(b_{i}\right)_{i \in[N]} \mid \sum_{i=1}^{N} b_{i} \alpha_{i}=\boldsymbol{a}\right\} & =\sum_{f_{H}}\left(\frac{a_{1}+\cdots+a_{k-1}}{c}+a_{j}+1\right) \\
& =Q^{\prime}(\boldsymbol{a}) \sum_{f_{H}} 1=Q^{\prime}(\boldsymbol{a}) \#\left\{\left(b_{i}\right)_{i \in[N-1]} \mid \sum_{i=1}^{N-1} b_{i} \alpha_{i}=\boldsymbol{a}\right\},
\end{aligned}
$$

where the first equality uses (15) and (16), and the last equality uses (14). 


\section{Type $C_{n+1}$ Kostant partition functions and the Baldoni-Vergne identities}

We now show two generalizations of Theorem 1 in the type $C_{n+1}$ case. We first give the necessary definitions and explain the notion of flow in the context of signed graphs. Throughout this section, the graphs $G$ on the vertex set $[n+1]$ we consider are signed, that is there is a sign $\epsilon \in\{+,-\}$ assigned to each of its edges, with possible multiple edges, and all loops labeled positive. Denote by $(i, j,-)$ and $(i, j,+), i \leq j$, a negative and a positive edge, respectively. Denote by $m_{i j}^{\epsilon}$ the multiplicity of edge $(i, j, \epsilon)$ in $G, i \leq j, \epsilon \in\{+,-\}$. To each edge $(i, j, \epsilon), i \leq j$, of $G$, associate the positive type $C_{n+1} \operatorname{root} \mathrm{v}(i, j, \epsilon)$, where $\mathrm{v}(i, j,-)=e_{i}-e_{j}$ and $\mathrm{v}(i, j,+)=e_{i}+e_{j}$. Let $\left\{\left\{\alpha_{1}, \ldots, \alpha_{N}\right\}\right\}$ be the multiset of vectors corresponding to the multiset of edges of $G$ as described above. Note that $N=\sum_{1 \leq i<j \leq n+1}\left(m_{i j}^{-}+m_{i j}^{+}\right)$. The Kostant partition function $K_{G}$ evaluated at the vector $\boldsymbol{a} \in \mathbb{Z}^{n+1}$ is defined as

$$
K_{G}(\boldsymbol{a})=\#\left\{\left(b_{i}\right)_{i \in[N]} \mid \sum_{i \in[N]} b_{i} \alpha_{i}=\boldsymbol{a} \text { and } b_{i} \in \mathbb{Z}_{\geq 0}\right\} .
$$

That is, $K_{G}(\boldsymbol{a})$ is the number of ways to write the vector $\boldsymbol{a}$ as a nonnegative linear combination of the positive type $C_{n+1}$ roots corresponding to the edges of $G$, without regard to order.

Just like in the type $A_{n}$ case, we would like to think of the vector $\left(b_{i}\right)_{i \in[N]}$ as a flow. For this we here give a precise definition of flows in the type $C_{n+1}$ case, of which type $A_{n}$ is of course a special case.

Let $G$ be a signed graph on the vertex set $[n+1]$. Let $\left\{\left\{e_{1}, \ldots, e_{N}\right\}\right\}$ be the multiset of edges of $G$, and $\left\{\left\{\alpha_{1}, \ldots, \alpha_{N}\right\}\right\}$ the multiset of vectors corresponding to the multiset of edges of $G$. Fix an integer vector $\boldsymbol{a}=\left(a_{1}, \ldots, a_{n}, a_{n+1}\right) \in \mathbb{Z}^{n+1}$. A nonnegative integer a-flow $\boldsymbol{f}_{G}$ on $G$ is a vector $\boldsymbol{f}_{G}=\left(b_{i}\right)_{i \in[N]}\left(b_{i} \in \mathbb{Z}_{\geq 0}\right)$ such that for all $1 \leq i \leq n+1$, we have

$$
\sum_{\substack{e \in E \\ \operatorname{inc}(e, v)=-}} b(e)+a_{v}=\sum_{\substack{e \in E \\ \operatorname{inc}(e, v)=+}} b(e)+\sum_{e=(v, v,+)} b(e),
$$

where $b\left(e_{i}\right)=b_{i}$, inc $(e, v)=-$ if edge $e=(g, v,-), g<v$, and inc $(e, v)=+$ if $e=(g, v,+), g<v$, or $e=(v, j, \epsilon), v<j$, and $\epsilon \in\{+,-\}$.

Call $b(e)$ the flow assigned to edge $e$ of $G$. If the edge $e$ is negative, one can think of $b(e)$ units of fluid flowing on $e$ from its smaller to its bigger vertex. If the edge $e$ is positive, then one can think of $b(e)$ units of fluid flowing away both from $e$ 's smaller and bigger vertex to infinity. Edge $e$ is then a "leak" taking away $2 b(e)$ units of fluid.

From the above explanation it is clear that if we are given an $\boldsymbol{a}$-flow $\boldsymbol{f}_{G}$ such that $\sum_{e=(i, j,+), i \leq j} b(e)=y$, then $\boldsymbol{a}=\left(a_{1}, \ldots, a_{n}, 2 y-\sum_{i=1}^{n} a_{i}\right)$. It is then a matter of checking the definitions to see that for a signed graph $G$ on the vertex set $[n+1]$ and 
vector $\boldsymbol{a}=\left(a_{1}, \ldots, a_{n}, 2 y-\sum_{i=1}^{n} a_{i}\right) \in \mathbb{Z}^{n+1}$, the number of nonnegative integer $\boldsymbol{a}$-flows on $G$ is equal to $K_{G}(\boldsymbol{a})$.

Thinking of $K_{G}(\boldsymbol{a})$ as the number of nonnegative integer $\boldsymbol{a}$-flows on $G$, there is a straightforward generalization of Theorem 1 in the type $C_{n+1}$ case:

Theorem 3. Given a connected signed graph $G$ on the vertex set $[n+1]$ with $m_{n-1, n}^{-}=m_{n-1, n+1}^{-}=m_{n, n+1}^{-}=1, m_{j, n-1}^{+}=m_{j, n}^{+}=m_{j, n+1}^{+}=0$, for $j \in[n+1]$, and such that

$$
\frac{m_{j, n-1}^{-}+m_{j, n}^{-}+m_{j, n+1}^{-}}{m_{j, n-1}^{-}}=c \quad \text { for all } j \in[n-2],
$$

for some constant $c$ independent of $j$, we have

$$
K_{G}(\boldsymbol{a})=Q^{\prime \prime}(\boldsymbol{a}) K_{G-(n-1, n)}(\boldsymbol{a})
$$

for any $\boldsymbol{a}=\left(a_{1}, \ldots, a_{n}, 2 y-\sum_{i=1}^{n} a_{i}\right) \in \mathbb{Z}^{n+1}$, where

$$
Q^{\prime \prime}(\boldsymbol{a})=\frac{a_{1}+\cdots+a_{n-2}-2 y}{c}+a_{n-1}+1 \text {. }
$$

The proof of Theorem 3 proceeds analogously to that of Theorem 1. Namely, define partial flows $\boldsymbol{f}_{H}=\left(b_{i}\right)_{i \in[N-3]}$ on

$$
H:=G-\{(n-1, n,-),(n-1, n+1,-),(n, n+1,-)\}
$$

such that

$$
\sum_{i=1}^{N-3} b_{i} \alpha_{i}=\left(a_{1}, \ldots, a_{n-2}, x_{n-1}, x_{n}, x_{n+1}\right),
$$

for some $x_{n-1}, x_{n}, x_{n+1} \in \mathbb{Z}$, such that $x_{n-1} \leq a_{n-1}, x_{n} \leq a_{n}$ and the sum of flows on positive edges is $y$.

Then, one can prove:

- The elements of the set partial flows are in bijection with the nonnegative integer $\boldsymbol{a}$-flows on $G-(n-1, n)$. That is,

$$
\text { \# partial flows }=K_{G-(n-1, n)}(\boldsymbol{a}) .
$$

- The elements of the multiset of partial flows $f_{H}$, whose cardinality is $Q^{\prime \prime}(\boldsymbol{a})$ times the cardinality of the set of partial flows, are in bijection with the nonnegative integer $\boldsymbol{a}$-flows on $G$. That is,

$$
Q^{\prime \prime}(\boldsymbol{a})(\# \text { partial flows })=K_{G}(\boldsymbol{a}) .
$$

Thus,

$$
K_{G}(\boldsymbol{a})=Q^{\prime \prime}(\boldsymbol{a}) K_{G-(n-1, n)}(\boldsymbol{a}) .
$$


We do not have to require that only negative edges are incident to the vertices $n-1, n, n+1$ in $G$, as the following theorem shows. The proof is analogous to earlier ones.

Theorem 4. Given a connected signed graph $G$ on the vertex set $[n+1]$ with $m_{n-1, n}^{-}=m_{n-1, n+1}^{-}=m_{n, n+1}^{-}=1, m_{i, j}^{+}=0$, for $i, j \in\{n-1, n, n+1\}$, and such that

$$
\frac{m_{j, n-1}^{\epsilon}+m_{j, r}^{\epsilon}+m_{j, n+1}^{\epsilon}}{m_{j, n-1}^{\epsilon}}=c \quad \text { for all } j \in[n-2] \text { and } \epsilon \in\{+,-\},
$$

for some constant $c$ independent of $j$, we have

$$
K_{G}(\boldsymbol{a})=\left(\frac{a_{1}+\cdots+a_{n-2}-2 y}{c}+a_{n-1}+1\right) K_{G-(n-1, n)}(\boldsymbol{a})
$$

for any $\boldsymbol{a}=\left(a_{1}, \ldots, a_{n}, 2 y-\sum_{i=1}^{n} a_{i}\right) \in \mathbb{Z}^{n+1}$.

Of course, Theorem 2 also has type $C_{n+1}$ generalizations and variations. We invite the reader to write these out and check each step of the proof of Theorem 1 and see how they can be adapted.

\section{Acknowledgement}

I thank Richard Stanley for his intriguing slides and for pointing out the work of Baldoni and Vergne. I also thank Alejandro Morales and Alex Postnikov for numerous conversations about flows and the Kostant partition function.

\section{References}

[Baldoni and Vergne 2008] W. Baldoni and M. Vergne, "Kostant partitions functions and flow polytopes,” Transform. Groups 13:3-4 (2008), 447-469. MR 2009k:52029 Zbl 1200.52008

[Stanley 2000] R. P. Stanley, “Acyclic flow polytopes and Kostant's partition function," conference transparencies, 2000, Available at http://www-math.mit.edu/ rstan/transparencies/kostant.ps.

Received November 27, 2011. Revised April 4, 2012.

KAROLA MÉSZÁROS

DEPARTMENT OF MATHEMATICS

CORNELL UNIVERSITY

ITHACA, NY 14853

UNITED STATES

karola@math.cornell.edu 


\title{
PACIFIC JOURNAL OF MATHEMATICS
}

\author{
http://pacificmath.org \\ Founded in 1951 by \\ E. F. Beckenbach (1906-1982) and F. Wolf (1904-1989)
}

\section{EDITORS}

V. S. Varadarajan (Managing Editor)

Department of Mathematics

University of California

Los Angeles, CA 90095-1555

pacific@math.ucla.edu

Vyjayanthi Chari

Department of Mathematics

University of California

Riverside, CA 92521-0135

chari@math.ucr.edu

\section{Robert Finn}

Department of Mathematics Stanford University

Stanford, CA 94305-2125

finn@math.stanford.edu

Kefeng Liu

Department of Mathematics

University of California

Los Angeles, CA 90095-1555

liu@math.ucla.edu
Darren Long

Department of Mathematics

University of California

Santa Barbara, CA 93106-3080

long@math.ucsb.edu

Jiang-Hua Lu

Department of Mathematics

The University of Hong Kong

Pokfulam Rd., Hong Kong jhlu@maths.hku.hk

Alexander Merkurjev

Department of Mathematics

University of California

Los Angeles, CA 90095-1555

merkurev@math.ucla.edu
Sorin Popa

Department of Mathematics University of California

Los Angeles, CA 90095-1555 popa@math.ucla.edu

Jie Qing

Department of Mathematics

University of California

Santa Cruz, CA 95064

qing@cats.ucsc.edu

Jonathan Rogawski

Department of Mathematics

University of California

Los Angeles, CA 90095-1555

jonr@math.ucla.edu

\section{PRODUCTION}

pacific@math.berkeley.edu

\section{SUPPORTING INSTITUTIONS}

ACADEMIA SINICA, TAIPEI

CALIFORNIA INST. OF TECHNOLOGY INST. DE MATEMÁTICA PURA E APLICADA KEIO UNIVERSITY

MATH. SCIENCES RESEARCH INSTITUTE NEW MEXICO STATE UNIV.

OREGON STATE UNIV.

\author{
STANFORD UNIVERSITY \\ UNIV. OF BRITISH COLUMBIA \\ UNIV. OF CALIFORNIA, BERKELEY \\ UNIV. OF CALIFORNIA, DAVIS \\ UNIV. OF CALIFORNIA, LOS ANGELES \\ UNIV. OF CALIFORNIA, RIVERSIDE \\ UNIV. OF CALIFORNIA, SAN DIEGO \\ UNIV. OF CALIF., SANTA BARBARA
}

\author{
UNIV. OF CALIF., SANTA CRUZ \\ UNIV. OF MONTANA \\ UNIV. OF OREGON \\ UNIV. OF SOUTHERN CALIFORNIA \\ UNIV. OF UTAH \\ UNIV. OF WASHINGTON \\ WASHINGTON STATE UNIVERSITY
}

These supporting institutions contribute to the cost of publication of this Journal, but they are not owners or publishers and have no responsibility for its contents or policies.

See inside back cover or pacificmath.org for submission instructions.

The subscription price for 2012 is US \$420/year for the electronic version, and \$485/year for print and electronic.

Subscriptions, requests for back issues from the last three years and changes of subscribers address should be sent to Pacific Journal of Mathematics, P.O. Box 4163, Berkeley, CA 94704-0163, U.S.A. Prior back issues are obtainable from Periodicals Service Company, 11 Main Street, Germantown, NY 12526-5635. The Pacific Journal of Mathematics is indexed by Mathematical Reviews, Zentralblatt MATH, PASCAL CNRS Index, Referativnyi Zhurnal, Current Mathematical Publications and the Science Citation Index.

The Pacific Journal of Mathematics (ISSN 0030-8730) at the University of California, c/o Department of Mathematics, 969 Evans Hall, Berkeley, CA 94720-3840, is published monthly except July and August. Periodical rate postage paid at Berkeley, CA 94704, and additional mailing offices. POSTMASTER: send address changes to Pacific Journal of Mathematics, P.O. Box 4163, Berkeley, CA 94704-0163.

PJM peer review and production are managed by EditFLOW ${ }^{\mathrm{TM}}$ from Mathematical Sciences Publishers.

PUBLISHED BY PACIFIC JOURNAL OF MATHEMATICS

at the University of California, Berkeley 94720-3840

A NON-PROFIT CORPORATION

Typeset in LATEX

Copyright $(02012$ by Pacific Journal of Mathematics 


\section{PACIFIC JOURNAL OF MATHEMATICS}

Volume $260 \quad$ No. $1 \quad$ November 2012

The decomposition of global conformal invariants: Some technical proofs II

SPYROS ALEXAKIS

On deformation quantizations of hypertoric varieties

GWYN BELLAMY and TOSHIRO KUWABARA

Almost factoriality of integral domains and Krull-like domains

GYU Whan CHANG, HWANKOO KIM and JUNG WOOK LIM

Singularities of free group character varieties

CARLOS FLORENTINO and SEAN LAWTON

Energy identity for the maps from a surface with tension field bounded 181 in $L^{p}$

LI JIAYU and ZHU XIANGRONG

Remarks on some isoperimetric properties of the $k-1$ flow

YU-CHU LIN and DONG-Ho TSAI

Demystifying a divisibility property of the Kostant partition function 215

KAROLA MÉSZÁros

Exceptional Lie algebras, SU(3), and Jordan pairs

PIERO TRUINI

Lower estimate of Milnor number and characterization of isolated homogeneous hypersurface singularities

Stephen S.-T. Yau and HuAiqing Zuo 